\title{
Prophylactic ankle supports effects on time to stabilization, perceived stability and ground reaction force during lateral landing in female collegiate athletes with chronic ankle instability
}

Ali Yalfani ${ }^{1}$ and Zahra Raeisi ${ }^{*}$

\begin{abstract}
Background: This study was designed to investigate effects of Kinesiotape (KT) with closed basket weave method and lace-up braces (LB) on the vertical time to stabilization, peak vertical ground reaction force (PvGRF), and time to PVGRF as well as perceived stability during lateral landing of participants with chronic ankle instability before and after fatigue.

Methods: Thirty female college athletes with chronic ankle instability of three conditions (control, KT, and LB) performed lateral landing from a $30 \mathrm{~cm}$ high step on the plantar pressure platform pre and post fatigue.

Results: The pre-test findings on the rearfoot, of $L B$ indicated negatively increased the PVGRF force $\left(F_{2,58}=3.63, P=\right.$ 0.04) and decreased the time to $\operatorname{PvGRF}\left(F_{2,58}=4.67, P=0.01\right)$. The Bonferroni post-hoc testing revealed $L B$ condition increased the PVGRF than the control $(P=0.002)$ and $K T(P=0.038)$. Also, the post-hoc testing showed $L B$ condition decreased the time to PVGRF force than the control $(P=0.05)$ and $K T(P=0.01)$. The $L B$ negatively prolonged vertical time to stabilization in the forefoot $\left(F_{2,58}=6.74, P=0.002\right)$ and rearfoot $\left(F_{2,58}=6.13, P=0.004\right)$ after fatigue. The post-hoc testing revealed $L B$ condition generated a slower vertical time to stabilization than the control and $K T$ conditions $(P \leq 0.05)$. The use of $K T$ had no positive effects as elevated the PVGRF in the forefoot post fatigue $\left(F_{2,58}=\right.$ 7.11, $P=0.002)$. The post-hoc test uncovered that $K T$ augmented the PVGRF than control $(P=0.01)$ and $L B(P<$ 0.001). On the other hand, using $K T$ had psychological effects at pre-fatigue which resulting significantly greater in perceived stability compared to other conditions $\left(F_{2,58}=9.65, P<0.001\right)$. The post-hoc test showed that using $\mathrm{KT}$ increased perceived stability than LB $(P=0.004)$ and control $(P<0.001)$. Moreover, perceived stability improved significantly in $K T$ and $L B$ compared to the control condition at the post-fatigue $(P \leq 0.001)$.

Conclusions: Despite the positive psychological impact of the prophylactic ankle supports, there were no positive effect on the vertical time to stabilization, PvGRF, and time to PvGRF. Further studies are needed to distinguish the psychological and actual effects of prophylactic ankle supports on athletes with chronic ankle instability.
\end{abstract}

Keywords: Kinesiotape, Lace-up brace, Lateral landing, Dynamic control

\footnotetext{
* Correspondence: Z_raisi13@yahoo.com

${ }^{2}$ Department of Sports Rehabilitation, Faculty of Sport Science, Arak University, Arak, Iran

Full list of author information is available at the end of the article
}

C C The Author(s). 2021 Open Access This article is licensed under a Creative Commons Attribution 4.0 International License, which permits use, sharing, adaptation, distribution and reproduction in any medium or format, as long as you give appropriate credit to the original author(s) and the source, provide a link to the Creative Commons licence, and indicate if changes were made. The images or other third party material in this article are included in the article's Creative Commons licence, unless indicated otherwise in a credit line to the material. If material is not included in the article's Creative Commons licence and your intended use is not permitted by statutory regulation or exceeds the permitted use, you will need to obtain permission directly from the copyright holder. To view a copy of this licence, visit http://creativecommons.org/licenses/by/4.0/ The Creative Commons Public Domain Dedication waiver (http://creativecommons.org/publicdomain/zero/1.0/) applies to the data made available in this article, unless otherwise stated in a credit line to the data. 


\section{Background}

Lateral ankle sprains (LASs) are the most prevalent musculoskeletal injuries that occur among physically active individuals [1]. Such an injury is accompanied by a number of adverse effects, including significant athletic treatment costs; prolonged pain; absence from sport, work, and school; increased healthcare costs; decrease quality of life; increased odds ratio of recurrent ankle sprain; and premature arthritis [1-4]. Its high prevalence rate and resulting side effects have highlighted the need for specialists to accord urgent priority to the development of various prophylactic strategies intended to reduce the extent of ankle sprain injury. Current protective measures against the risk of ankle injury often encompass strength training for leg muscles, proprioceptive training, and the use of high-top shoes and prophylactic ankle supports (PASs) [5, 6].

PASs are classified mainly into ankle braces and tapes, each coming in different variants. They can effectively protect the ligament structure to prevent of ankle sprain by providing mechanical support and enhancing proprioception sense [7]. This prophylactic equipment is therefore frequently used by athletes with and without a previous history of LAS to prevent serious injury $[1,8]$.

Different injuries are caused by exercise activities and dominant movements in sports, among other factors. Volleyball and basketball, for example, account for 86 and $58 \%$ of ankle injuries that occur during landing, respectively [9]. These physical traumas are potentially affected by the magnitude of the ground reaction force (GRF) acting on the body as it performs a landing task and the need to maintain the body's perceived stability after initial foot contact with the ground [10]. A necessary requirement, then, is to minimize contact force during landing to reduce the force applied to ankle structures and thereby decrease damage to the ankles as the only body part that comes in contact with the ground during landing [8]. An athlete's ability to effectively lessen landing effects is determined by the vertical GRF (vGRF) [11]' whose peak value (i.e., PvGRF) has been investigated as a determinant of injury given that the large forces occurring at this moment are associated with injury or instability when combined with joint malalignment [12]. Another indicator explored in research is time to stabilization (TTS), which points to the ability of an individual to restore balance in a state wherein a shift in condition from dynamic to static motion occurs on the base of support [13]. The ability to stabilize rapidly after landing is considered a positive or prophylactic feature because injury mitigation is influenced by the effective maintenance of the body's perceived stability after landing [12]. Finally, ankle injury can also be caused by fatigue. According to Tropp et al., more than $40 \%$ of ankle injuries occur near the end of an activity and post-fatigue [14]. Fatigue reduces afferent inputs, decreases the ability to respond quickly to proprioceptive feedback, and ultimately increases postural oscillations and affects postural control [15, 16]. Fatigue is also one of the factors that directly affects vGRF and TTS after landing [17].

Although athletes frequently use PASs as protection against physical trauma, studies have presented contrasting results on PAS usage and their impact on GRF, time to peak GRF, and TTS. Some researchers, for instance, showed that the use of Kinesio tape differentially affects TTS and GRF [3, 6, 7, 9, 10, 18-20]. Hunt and Short found that taping increases self-esteem and reduces anxiety due to injury or re-injury [21]. In these studies, as well, the failure of PASs to influence participant performance and compare actual and psychological effects motivated researchers to put forward a placebo effect theory in regard to these instruments [22, 23].

Landing has been recognized as one of the most common causes of non-contact ankle sprain [24], with the direction of landing shown as an influencing factor for dynamic postural stability. In this respect, Liu et al. underscored the importance of perceived multidimensional stability tasks and suggested that lateral movements cause greater ankle injuries than do forward and vertical movements [13]. Despite the insights provided by such research, however, no study has been devoted to the effects of PASs on lateral landing before and after fatigue. This deficiency is worrisome because extant research involving forward jump landing tasks is insufficient to evaluate the causes and prevention of lower limb injuries. To fill this void, the current work conducted an experiment using lateral landing that imposes stress on the lateral and vulnerable parts of the ankle [13, 17]. Because the role of PASs in lateral landing and their effects on PvGRF, time to PvGRF, vertical TTS (vTTS), and perceived stability may differ from those of forward landing, this study investigated the effects of Kinesiotape (KT) and lace-up brace (LB) usage on the aforementioned variables before and after fatigue, with focus on female college student athletes with chronic ankle instability (CAI).

\section{Methods \\ Participants}

Thirty female collegiate athletes with CAI (mean age $=$ $22.86 \pm 2.01$ years, mean mass $=56.76 \pm 5.36 \mathrm{~kg}$, mean height $=164.32 \pm 4.4 \mathrm{~cm}$, mean score of Cumberland Ankle Instability Tool $($ CAIT $)=20.2 \pm 1.4)$ volunteered to participate in the study. The sample size was calculated by using GPower software, which was configured to run power analysis for a Cohen's d effect size of 0.4, with alpha level of 0.05 , and a test power of 0.95 . A final 
sample of 30 was established to consider dropouts. The inclusion criteria, which were based on the standards approved by the International Ankle Consortium, were as follows: more than two incidences of ankle sprains or a greater need for medical treatment of the condition, feelings of fear and instability in ankle function, the collapse of the foot during physical activities, a score of $\leq 24$ in the CAIT, and the confirmation of CAI via anterior drawer and talar tilt tests performed by an experienced physician [25]. The athletes recruited for participation should have had CAI on one foot only, with the affected leg as the dominant one. The participants were no history of ankle or knee injury in the past 3 months, as well as no history of surgery or fractures of the lower extremities; chronic diseases, such as patellofemoral pain syndrome; or apparent deformities, such as flat foot or high arch. The aim and procedures of the study were explained to the participants, who reviewed and voluntarily signed an informed written consent form. This project was approved by the local Ethics Committee (code IR.BASU.REC.1399.027) and also it was registered with an Iranian Registry of Clinical Trial (code IRCT20200204046368N3) and was performed according the Declaration of Helsinki.

\section{Instrumentation}

A plantar pressure platform (FDM-S, Zebris Medical $\mathrm{GmbH}$, Germany, $120 \mathrm{~Hz}$ acquisition frequency) composed of 2560 high-sensitivity sensors was used to record PvGRF, time to PvGRF, and vTTS during lateral landing.

\section{Experimental procedures}

All participants were asked to report to the Sport Rehabilitation Research Laboratory for a single occasion. First, each participant performed warm-up exercises for $10 \mathrm{~min}$.

The participant was tested under three conditions: The test limb was outfitted with a lace-up ankle brace (Model 4007, Oppo, USA) (Fig. 1a), wrapped in KT (Ares, Korea) in a complete closed basket weave pattern [26] (Fig. 1b), and left without a PAS (control). All the intervention applied by one certified athletic trainer to participants. The subject was then asked to perform a drop lateral landing task from a $30 \mathrm{~cm}$ high step that was positioned $15 \mathrm{~cm}$ from the center of a plantar pressure platform. To ensure that the task was correctly executed, the subject was instructed to place her nondominant leg on the step while fully supporting her body weight. The participant was subsequently prompted to carry out drop lateral landing while looking forward without putting weight on the test limb as she landed on the platform and maintain her balance for $10 \mathrm{~s}$ after landing.

Each participant underwent three practice trials before the identification of the correct task was initiated. All of them performed three lateral landing tasks on the test limb under the three aforementioned conditions before and after fatigue and were wearing the same type of shoes (Air Max, Nike). Each participant attended in 3 testing session which in each session 1 of 3 conditions (KT, LB and control) was performed. A minimum of 48 and a maximum of $96 \mathrm{~h}$ of rest were given between sessions to all subjects [10]. The sequence of the tests (LB,
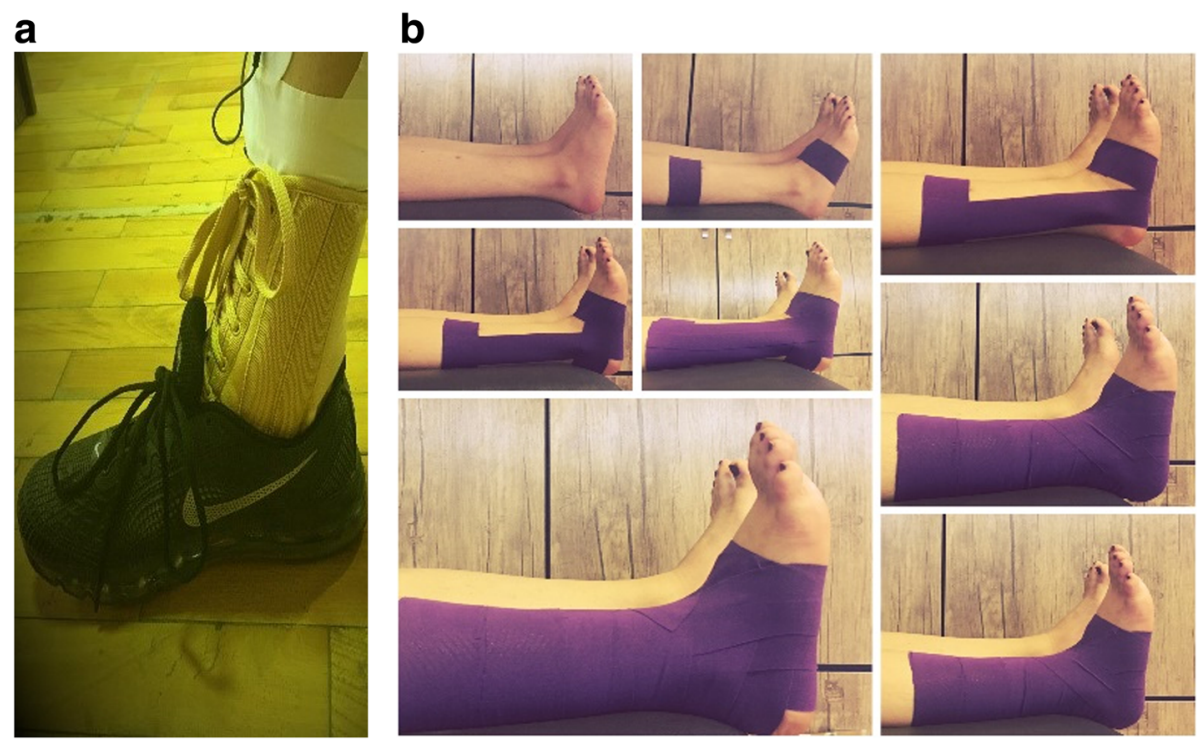

Fig. 1 a. Sample of ankle lace-up brace. $\mathbf{b}$. Sample of ankle closed basket weave pattern 
$\mathrm{KT}$, control) was determined by the participants randomly by lot.

\section{Fatigue protocol}

The Bruce protocol was performed to induce fatigue by using $\mathrm{h} / \mathrm{p} / \mathrm{cosmos}$ mercury med treadmill. The Borg Rating of Perceived Exertion and Polar Pacemaker were used to determine time to fatigue and control heart rate, respectively. The fatigue protocol was terminated when the subjects reached a score of 17 on the Borg scale and registered $80 \%$ of their maximum heart rate (age subtracted from 220). The subjects then performed the cool down phase for two minutes at their chosen speed. Immediately after fatigue, all the tests were repeated as part of the post-test.

\section{Psychological measure}

A four-point Likert scale (1: very unstable, 2: unstable, 3: stable, 4: very stable) was used to assess the psychological effects of the examined PASs [22]. The subjects were requested to use this scale to indicate their perception of stability under each lateral landing condition before and after fatigue. They were prohibited from going over their prior responses to avoid influence from previously acquired scores. After completing all the tests, the subjects were asked to answer the following question: "If given a choice, which among the three conditions-the use of KT, outfitting with a LB, or the control condition-would you prefer to adopt during engagement in sports activities?"

\section{Data processing}

Baseline vGRF data were obtained separately for the forefoot (two-thirds of foot length) and rearfoot (one- third of foot length) in each test using WinFDM-S software (Fig. 2). The trial data were then exported from the software, and the vTTS in each test was obtained using MATLAB (version R2010b, MathWorks, Natick, MA) following the procedure of Wright et al. [12]. Briefly, the data imported into MATLAB software were initially rectified and filtered with a $12 \mathrm{~Hz}$ second-order low-pass Butterworth filter. A normalized reference variable was calculated on the basis of the participants' trial results; that is, the mean vGRF in the last two seconds of each trial $(8-10 \mathrm{~s})$ was ascertained. Subsequently, three standard deviations from the mean for a range of normal variations were calculated for each participant in all the trials. An unbounded third ordinal polynomial with a rectified force of $10 \mathrm{~s}$ after landing was fitted for each participant and each trial. The vTTS was defined as the point at which the unbounded third ordinal polynomial exceeded the range of variation occurring in the first trial (Fig. 3). The vTTS of the forefoot and rearfoot was calculated for each participant in the different test conditions.

PvGRF and time to PvGRF values were calculated in each test. Given that the test output was separately generated by software for the forefoot and rearfoot, the PvGRF and time to PvGRF derived on the basis of the forefoot and rearfoot data were labeled PvGRF1, time to PvGRF1, PvGRF2, and time to PvGRF2, respectively.

\section{Statistical analysis}

The means and standard errors of the participants during the three lateral landing trials under each condition pre- and post-fatigue were subjected to statistical analysis. Four separate repeated-measures analyses of variance with two within-subject factors (condition and

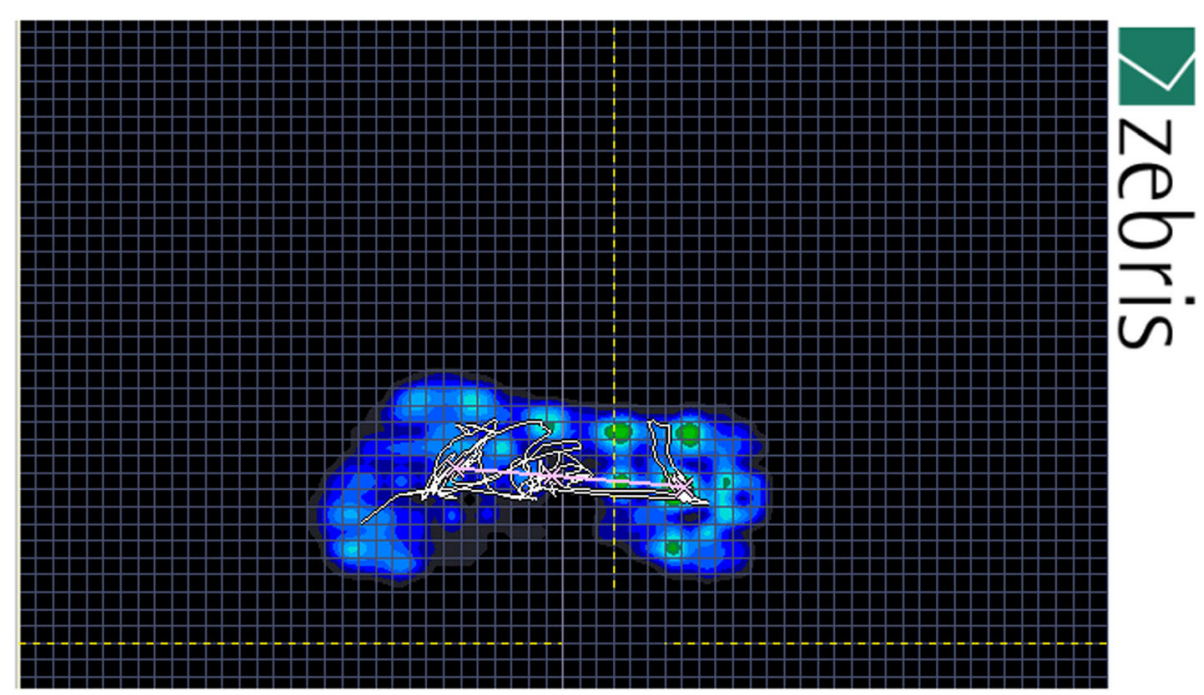

Fig. 2 WinFDM-S software sample data 


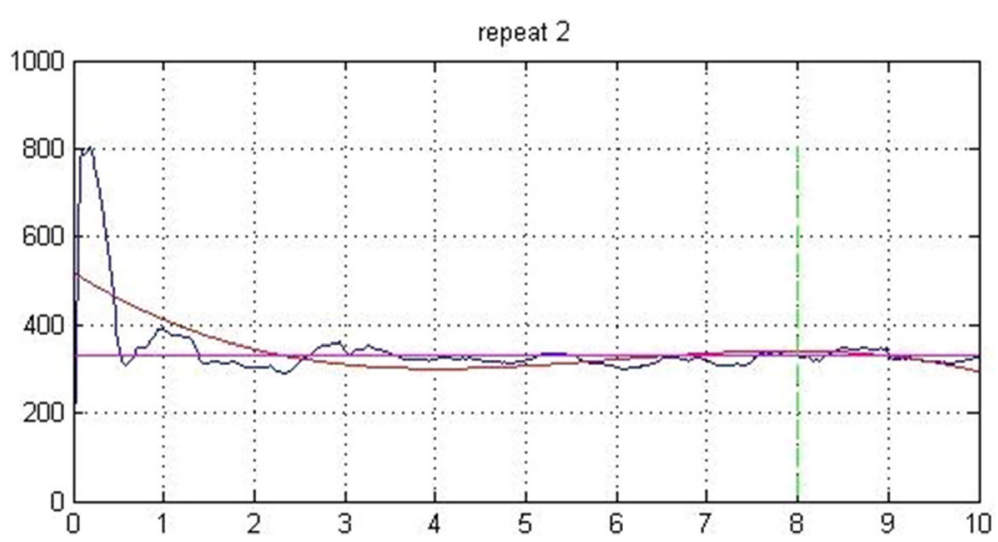

Fig. 3 Example of calculation of vertical time to stabilization of 1 participant

time) were conducted on PvGRF, time to PvGRF, vTTS, and perceived stability (dependent variables). A p value of 0.05 was considered statistically significant. The Bonferroni post-hoc test was carried out to determine statistically significant interactions, and the Statistical Package for the Social Sciences (version 21) was used to perform all the analyses.

\section{Results}

Tables 1,2 and 3 present the $\mathrm{M} \pm \mathrm{SD}$ of the studied variables.

\section{VTTS in the forefoot and rearfoot}

A condition-by-time interaction $\left(\mathrm{F}_{2,58}=6.74, P=0.002\right.$, effect size $=0.2$ ) occurred in relation to vTTS in forefoot. The post-hoc Bonferroni test revealed that outfitting with a LB resulted in a slower vTTS than that observed under the control $(P=0.01)$ and KT $(P=0.008)$ conditions after fatigue onset. The interaction between condition and time for vTTS in the rearfoot was significant $\left(\mathrm{F}_{2,58}=6.13, P=0.004\right.$, effect size $\left.=0.19\right)$. The Bonferroni post-hoc test results indicated that being fitted with a
LB generated a slower vTTS than that produced under the control $(P=0.009)$ and KT $(P=0.01)$ conditions post-fatigue.

\section{Perceived stability}

The interaction between condition and time with respect to perceived stability $\left(\mathrm{F}_{2,58}=9.65, P<0.001\right.$, effect size $=$ 0.27 ) was statistically significant. The post-hoc test showed that at pre-fatigue, using KT increased perceived stability to a level higher than that registered under the LB $(P=0.004)$ and control $(P<0.001)$ conditions. However, the latter two conditions generated no significant difference in perceived stability. The Bonferroni posthoc test also showed that at post-fatigue, the use of KT increased perceived stability to a level greater than that observed under the other two conditions. After fatigue, fitting with a LB elevated perceived stability to a degree higher than that occurring under the controls $(P<$ $0.001)$. In response to the general question about preference for landing conditions, 23 participants favored using Kinesio tape, and seven chose lace-up braces.
Table $1 \mathrm{M} \pm \mathrm{SD}$ of variables examined in relation to the forefoot

\begin{tabular}{cllll}
\hline Condition & Time & \multicolumn{3}{l}{$\mathbf{M} \pm$ SD variables } \\
\cline { 3 - 5 } & & $\mathbf{v T T S}(\mathbf{s})$ & PvGRF(N/cm $\left.{ }^{2}\right)$ & TT PvGRF (ms) \\
\hline Fore foot & & & & \\
Control & BF & $1.61 \pm 0.92$ & $984.02 \pm 295.48$ & $0.17 \pm 0.16$ \\
& AF & $1.44 \pm 0.93$ & $939.89 \pm 208.76$ & $0.12 \pm 0.03$ \\
Brace & BF & $1.99 \pm 1.3$ & $970.55 \pm 227.85$ & $0.26 \pm 0.46$ \\
& AF & $2.1 \pm 1.23$ & $909.23 \pm 220.27$ & $0.12 \pm 0.08$ \\
Tape & BF & $1.85 \pm 1.17$ & $936.04 \pm 220.34$ & $0.1 \pm 0.03$ \\
& AF & $1.02 \pm 0.57$ & $975.79 \pm 211.8$ & $0.1 \pm 0.02$ \\
\hline
\end{tabular}

Abbreviation: $B F$ Before fatigue, AF After fatigue, $v T T S$ Vertical time to stabilization, $v G R F$ Vertical ground reaction force, $\Pi T v G R F$ Time to vertical ground reaction force
Table $2 \mathrm{M} \pm \mathrm{SD}$ of variables examined in relation to the rearfoot

\begin{tabular}{|c|c|c|c|c|}
\hline \multirow[t]{2}{*}{ Condition } & \multirow[t]{2}{*}{ Time } & \multicolumn{3}{|c|}{$\mathrm{M} \pm \mathrm{SD}$ variables } \\
\hline & & $\overline{\text { vTTS(s) }}$ & $\operatorname{PvGRF}\left(\mathrm{N} / \mathrm{cm}^{2}\right)$ & TT PvGRF (ms) \\
\hline \multicolumn{5}{|l|}{ Rear foot } \\
\hline \multirow[t]{2}{*}{ Control } & $\mathrm{BF}$ & $1.36 \pm 1.05$ & $515.45 \pm 92.92$ & $1.04 \pm 1.57$ \\
\hline & AF & $1.24 \pm 1.12$ & $582.61 \pm 133.66$ & $0.85 \pm 1.15$ \\
\hline \multirow[t]{2}{*}{ Brace } & $\mathrm{BF}$ & $2.03 \pm 1.2$ & $620.01 \pm 182.48$ & $0.21 \pm 0.43$ \\
\hline & $\mathrm{AF}$ & $1.94 \pm 0.84$ & $582.7 \pm 169.46$ & $0.55 \pm 1.03$ \\
\hline \multirow[t]{2}{*}{ Tape } & $\mathrm{BF}$ & $1.74 \pm 1.09$ & $574.87 \pm 159.91$ & $1.29 \pm 2.23$ \\
\hline & $\mathrm{AF}$ & $0.98 \pm 0.94$ & $600.98 \pm 162.16$ & $0.83 \pm 1.08$ \\
\hline
\end{tabular}

Abbreviation: BF Before fatigue, AF After fatigue, $v T T S$ Vertical time to stabilization, $v G R F$ Vertical ground reaction force, $\Pi$ T $v R F m a x$ Time to vertical ground reaction force 
Table $3 \mathrm{M} \pm \mathrm{SD}$ of perceived stability score

\begin{tabular}{lll}
\hline Condition & Time & $\mathbf{M} \pm \mathbf{S D}$ \\
\hline Perception of stability & & \\
Control & BF & $3.2 \pm 0.32$ \\
& AF & $2.75 \pm 0.6$ \\
Brace & BF & $3.18 \pm 0.52$ \\
& AF & $3.36 \pm 0.48$ \\
Tape & BF & $3.72 \pm 0.47$ \\
& AF & $3.80 \pm 0.44$ \\
\hline
\end{tabular}

Abbreviation: $B F$ Before fatigue, $A F$ After fatigue

\section{PvGRF in the forefoot and rearfoot}

Statistical analysis of time interaction of PvGRF in the forefoot indicated significantly differences between the three conditions $\left(\mathrm{F}_{2,58}=7.11, P=0.002\right.$, effect size $\left.=0.21\right)$. The post-hoc test uncovered that at post-fatigue, using KT augmented the PvGRF at a degree exceeding that under the control $(P=0.01)$ and LB $(P<0.001)$ conditions. The condition-by-time interaction as regards PvGRF in the rearfoot also significantly differed across the interventions $\left(\mathrm{F}_{2,58}=3.63, P=0.04\right.$, effect size $\left.=0.12\right)$. The pre-fatigue LB condition increased the PvGRF to an extent greater than that occurring under the control $(P=0.002)$ and KT $(P=0.038)$ conditions.

\section{Time to PvGRF in the forefoot and rearfoot}

The results on the forefoot showed that no conditionby-time interaction as regards time to PvGRF occurred $\left(\mathrm{F}_{2,58}=2.63, P=0.1\right.$, effect size $\left.=0.08\right)$ but that such an interaction was statistically significant with respect to the rearfoot $\left(F_{2,58}=4.67, P=0.01\right.$, effect size $\left.=0.15\right)$. The Bonferroni post-hoc test results revealed that at prefatigue, the use of LB decreased the time to PvGRF to a level lower than that registered under the controls $(P=$ $0.05)$ and the use of KT $(P=0.01)$.

\section{Discussion}

The present study was designed to investigate effects of KT with closed basket weave method and LB on the vTTS, PvGRF, and time to PvGRF in the forefoot and rearfoot as well as perceived stability during lateral landing performed by female college athletes with CAI before and after fatigue.

\section{VTTS and psychological effects on perceived stability}

TTS is commonly examined in research on postural stability and the functioning of lower extremity joints, such as ankles and knees. It is defined as the time required for an individual to return to baseline values from an unstable position [27]. The results of this study showed that at pre-fatigue, the PASs had no effect on vTTS in the forefoot and rearfoot during lateral landing, whereas at post-fatigue, the use of LB negatively affected vTTS in the forefoot and rearfoot and generated a vTTS longer than that observed under the control and KT conditions. PASs are widely used among athletes to prevent injury, and the use of tape and braces has attracted the attention of many researchers $[2,3,6,10]$. Various studies on $\mathrm{KT}$ derived different effects, including increased skin blood flow during exercise, modified lymphatic circulation, support for ligaments and tendons, and a stimulated subcutaneous skin receptor mechanism; these effects enhance the activity of mechanoreceptors through a feedback mechanism and improve joint performance [5, 7]. Two broad theories have been used to explain the mechanism of brace efficacy: the first features passive mechanical support, and the second revolves around the improvement of sensorimotor function through increased stimulation of cutaneous receptors and joint mechanoreceptors [28].

Our hypothesis was that a brace model with support for lateral parts of the ankle can help control this body part during lateral landing and thus improve balance. Ankle stability was predicted to increase because of the eight-like shape and heel-lock structure used in the completed closed basket weave taping method. In spite of the advantages attributed to these PASs in the literature and in contrast to the hypothesis that we formulated, we did not observe a significant difference in vTTS during lateral landing with KT usage; meanwhile, at post-fatigue, the use of LB increased vTTS in the forefoot and rearfoot. Studies have shown that fatigue clearly affects the biomechanics of landing on one foot and that the risk of injury is greater after fatigue. Fatigue may alter neuromuscular control and diminish the body's ability to maintain stability $[6,11]$, thus driving the use of TTS in evaluating the impact of fatigue on proprioception and neuromuscular control. An increase in this variable indicates the body's delayed response to stability and difficulty in postural control during landing [6,27].

According to the results, LB usage did not improve vTTS and increased vTTS post-fatigue, which can increase the risk of injury. Brace weight potentially weakens postural control during fatigue and increases vTTS during lateral landing. Significant results were derived as regards psychological effects on the perceived stability of the participants. Unlike the LB and control conditions, KT usage resulted in greater perceived stability pre-fatigue. Such stability did not differ significantly under the LB and control conditions. Despite the increase in perceived stability during landing with KT use, the vTTS did not decrease significantly in this condition. Consistent with these results, Hunt and Short and Gear et al. reported an improvement in subjects' feelings of stability and self-confidence in performing functional tests with KT fitting, even though no significant difference was found in the participants' performance [21, 
22]. In our study, the participants felt that the ankle was more stable post-fatigue when they used a PAS in executing landing than when no support was employed. In addition to the tape, the LB also significantly differed from the controls in terms of improvement to perceived stability.

The vTTS results increased with LB usage during lateral landing. Sawkins et al. stated that if athletes believe that PASs protect them from injury, they may participate in an activity more confidently [23]. In the present work, the increase in vTTS with LB usage at post-fatigue may have enhanced the participants' sense of stability-an outcome that did not arise before the onset of fatigue. According to expectancy theory, athletes rely on the effects of PASs in preventing injury. As a result, inducing the belief that a placebo is effective and increasing perceived stability are easy, as asserted by Sawkins et al. [23]. However, the use of PASs seems to result in less precise lateral landing owing to the creation of a false feeling of safety and an increase in false self-confidence. This can increase the risk of injury, especially during fatigue, in more difficult situations, such as races and competitions or the performance of complex and highspeed tasks.

A notable finding in this work was that all the 30 subjects with CAI preferred using the PASs as supports in performing landing, with 23 favoring $\mathrm{KT}$ for the increased sense of ankle stability that it provided, and seven preferring to use LB.

\section{PvGRF and time to PvGRF}

At pre-fatigue, using LB increased PvGRF in the rearfoot but decreased the time to PvGRF. The use of KT augmented PvGRF in the forefoot during lateral landing after fatigue. PvGRF is a pivotal and desirable variable for evaluating landing because it eases measurement and generates accurate results [8]. It can also indicate an athlete's ability to effectively reduce landing effects. The lower PvGRF show the better landing strategy; strong force can lead to injuries to the ankle and knee joints [11]. PASs are primarily intended to limit the excessive inversion of the ankle and foot complex while allowing normal plantar flexion and dorsiflexion to maintain function. However, studies showed that the range of motion (ROM) of plantar flexion and dorsiflexion will also be limited when an ankle brace or tape is used $[3,7,10$, 29]. Decreasing the normal ROM of the ankle can affect the entire lower extremity and normal movement patterns and thereby weaken the body's ability to absorb energy upon landing and impose greater GRF on the body [10]. Damage to structures such as the subchondral bone, cartilage, and soft tissue may also occur as a result of increased GRF [3].
Studies demonstrated that the use of PASs not only influences the ROM of joints but also reduces muscle activity, which may diminish the auxiliary role of some muscles in minimizing body acceleration during landing [28]. Fatigue is an integral part of physical activity. When it occurs, reaction times against external stimuli are delayed, and the likelihood of injury increases [27]. This risk of injury is exacerbated on initial contact because the body at this stage cannot move within an ROM to allow contact forces to be absorbed through active structures (such as muscles) [27, 29]. Previous studies reported an increase in PvGRF in forward drop landing with decreasing joint ROM and leg muscle activity due to PAS use $[3,20,29]$. Our results also indicated that PvGRF increased before and after fatigue in rear foot under the use of LB and KT, respectively. Similarly, the use of KT increased PvGRF in the forefoot postfatigue. These results are consistent with those of Cordova et al. [29] and Distefano et al. [3] but inconsistent with those of Hodgson et al. [20] Note that the task performed in the present study was lateral landing, whereas that in most previous works was forward landing.

The findings likewise illustrated that ankle bracing reduced the time to PvGRF in the rearfoot to levels lower than those occurring under the control and KT conditions. The increasing PvGRF and decreasing time to PvGRF during lateral landing under LB usage suggested that under this condition, musculoskeletal structures are affected by greater loads imposed at a shorter time [10]. These changes also implied that the ankle's ability to absorb energy decreases when certain PASs are used; this effect, in turn, increases the load imposed on proximal joints, including the knee [10]. In this regard, Cordova et al. showed that some ankle stabilizers impair the optimal performance of this joint in absorbing contact by restricting the ROM of the ankle [29]. Our results are consistent with those derived by Henderson et al.[9] and Hodgson et al. [20] on the increase in PvGRF during landing with braces. They are also compatible with the findings of Riemann et al. [10] on the reduction of time to PvGRF.

Because the LB used in this study covers a large area of the soles of the foot and provides support to the lateral parts of the ankle, its use likely reduced the ROM of the rearfoot and may have decreased the time to PvGRF in the rearfoot by shortening its contact with the ground. Contrary to studies on forward landing drops that found a PvGRF2 larger than PvGRF1, the current research uncovered a PvGRF2 larger than PvGRF1 during lateral landing and a PvGRF1 greater than PvGRF2 at all landing conditions before and after fatigue.

The literature discussed the effects of general fatigue on balance, which is why a general fatigue protocol was used in the present study. Nevertheless, a different 
mechanism and effect may arise with regard to functional fatigue. Given that only a lateral landing test was performed on the injuried leg, no data on the other leg was derived for comparison in regard to PAS effects. Findings may also differ depending on task type. Finally, the same shoes were used by all the participants for matching in the test, but using other types of footwear may generate different results.

\section{Conclusions}

Our results showed that the examined PASs had no positive effect on vTTS, PvGRF, and time to PvGRF in the forefoot and rearfoot despite their positive psychological influence on the participants' perceived stability during lateral landing. Because the results obtained for the forefoot and rearfoot were separately accurate, PvGRF in the rearfoot was not necessarily larger than PvGRF in the forefoot during task performance. These values appear to be directly related to individual landing techniques and type of task performed.

Given that only lateral landing was examined in this work, the use of KT and LB in other tasks may produce varying outcomes. Further study should be directed toward distinguishing the actual and psychological effects of PASs specially after fatigue in other tasks.

\begin{abstract}
Abbreviations
LASs: Lateral ankle sprains; PASs: Prophylactic ankle supports; GRF: Ground reaction force; VGRF: Vertical ground reaction force; TTS: Time to stabilization; KT: Kinesiotape; LB: Lace-up brace; CAl: Chronic ankle instability; CAIT: Cumberland Ankle Instability Tool; PvGRF: peak vertical GRF; BF: Before fatigue; AF: After fatigue; vTTS: Vertical time to stabilization; $\Pi$ VGRF: Time to vertical ground reaction force; ROM: Range of motion
\end{abstract}

\section{Acknowledgements}

The authors would like to thank the participants that took part in this study.

\section{Authors' contributions}

All authors read and approved the final manuscript.

\section{Funding}

Not applicable.

\section{Availability of data and materials}

The datasets used and/or analyzed during the current study are available from the corresponding author on reasonable request.

\section{Declarations}

\section{Ethics approval and consent to participate}

This project was approved by the local Ethics Committee of Hamedan University of Medical Science (code IR.BASU.REC.1399.027) and also it was registered with an Iranian Registry of Clinical Trial (code

IRCT20200204046368N3). Before experimental procedures began, all the participants reviewed and voluntarily signed an informed written consent form.

\section{Consent for publication}

Not applicable.

\section{Competing interests}

The authors declare that they have no competing interests.

\section{Author details}

${ }^{1}$ Department of Sports Rehabilitation, Faculty of Sport Science, Bu-Ali Sina University, Hamedan, Iran. ${ }^{2}$ Department of Sports Rehabilitation, Faculty of Sport Science, Arak University, Arak, Iran.

Received: 5 March 2021 Accepted: 24 May 2021

Published online: 03 June 2021

\section{References}

1. Gribble PA. Evaluating and differentiating ankle instability. J Athl Train. 2019; 54:617-27.

2. Doherty C, Delahunt E, Caulfield B, Hertel J, Ryan J, Bleakley C. The incidence and prevalence of ankle sprain injury: A systematic review and meta-analysis of prospective epidemiological studies. Sports Medicine. 2014; 44:123-40.

3. DiStefano L, Padua DA, Brown CN, Guskiewicz KM. Lower extremity kinematics and ground reaction forces after prophylactic lace-up ankle bracing. J Athl Train. 2008;43(3):234-41. https://doi.org/10.4085/10626050-43.3.234.

4. Delahunt E, Remus A. Risk factors for lateral ankle sprains and chronic ankle instability. J Athl Train. 2019;54:611-6.

5. Briem K, Eythörsdöttir H, Magnúsdóttir RG, Pálmarsson R, RúnarsdÖttir T, Sveinsson T. Effects of Kinesio Tape Compared With Nonelastic Sports Tape and the Untaped Ankle During a Sudden Inversion Perturbation in Male Athletes. J Orthop Sport Phys Ther. 2011;41:328-35. https://doi.org/10.2519/ jospt.2011.3501.

6. Shaw MY, Gribble PA, Frye JL. Ankle bracing, fatigue, and time to stabilization in collegiate volleyball athletes. J Athl Train. 2008;43:164-71.

7. Botsis A, Schwarz N, Harper M, Liu W, Rooney C, Gurchiek L, et al. Effect of Kinesio ${ }^{\oplus}$ Taping on Ankle Complex Motion and Stiffness and Jump Landing Time to Stabilization in Female Ballet Dancers. J Funct Morphol Kinesiol. 2019;4:19. https://doi.org/10.3390/jfmk4020019.

8. Niu W, Feng T, Wang L, Jiang C, Zhang M. Effects of prophylactic ankle supports on vertical ground reaction force during landing: A meta-analysis. J Sport Sci Med. 2016:15:1-10.

9. Henderson ZJ, Sanzo P, Zerpa C, Kivi D. The Effects of Ankle Braces on Lower Extremity Electromyography and Performance During Vertical Jumping: A Pilot Study. Int J Exerc Sci. 2019;12:15-23. http://www.ncbi.nlm. nih.gov/pubmed/30761189.

10. Riemann BL, Schmitz RJ, Gale M, McCaw ST. Effect of ankle taping and bracing on vertical ground reaction forces during drop landings before and after treadmill jogging. J Orthop Sports Phys Ther. 2002;32:628-35.

11. Pappas $E$, Sheikhzadeh A, Hagins M, Nordin M. The effect of gender and fatigue on the biomechanics of bilateral landings from a jump: Peak values. J Sport Sci Med. 2007;6:77-84.

12. Wright CJ, Arnold BL, Ross SE. Altered kinematics and time to stabilization during drop-jump landings in individuals with or without functional ankle instability. J Athl Train. 2016;51:5-15.

13. Liu K, Heise GD. The effect of jump-landing directions on dynamic stability. J Appl Biomech. 2013;29:634-8. http://www.ncbi.nlm.nih.gov/pubmed/231 82979. Accessed 26 Jul 2019.

14. Tropp H, Ekstrand J, Gillquist J. Stabilometry in functional instability of the ankle andits value in predicting injury. Med Sci Sports Exerc. 1984;16:64-6.

15. Paillard T. Effects of general and local fatigue on postural control: A review. Neuroscience and Biobehavioral Reviews. 2012;36:162-76.

16. Webster KA, Pietrosimone BG, Gribble PA. Muscle Activation During Landing Before and After Fatigue in Individuals With or Without Chronic Ankle Instability. J Athl Train. 2016;51:629-36. https://doi.org/10.4085/1062-6050-51.10.01.

17. Malmir K, Olyaei GR, Talebian S, Jamshidi AA. Comparing the Effects of Peroneal Muscle Fatigue and Cyclic Loading on Ankle Neuromuscular Control During Lateral-Hop Landing. J Sport Rehabil. 2015;24:293-9. https:// doi.org/10.1123/jsr.2014-0165.

18. Fayson SD, Needle AR, Kaminski TW. The Effect of Ankle Kinesio Tape on Ankle Muscle Activity during a Drop Landing. J Sport Rehabil. 2015;24:3917. https://doi.org/10.1123/jsr.2014-0221.

19. Huang C-Y, Hsieh T-H, Lu S-C, Su F-C. Effect of the Kinesio tape to muscle activity and vertical jump performance in healthy inactive people. Biomed Eng Online. 2011;10:70. https://doi.org/10.1186/1475-925X-10-70.

20. Hodgson B, Tis L, Cobb S, Higbie E. The effect of external ankle support on vertical ground-reaction force and lower body kinematics. J Sport Rehabil. 2005;14:301-12. 
21. Hunt E, Short S. Collegiate athletes' perceptions of adhesive ankle taping: A qualitative analysis. J Sport Rehabil. 2006;15:280-98.

22. Gear W, Lundstrom M. Effect of Ankle Taping on Dynamic Balance and Perception of Stability. J Sport Med Allied Heal Sci Off J Ohio Athl Trainers' Assoc. 2018;4. doi:https://doi.org/10.25035/jsmahs.04.02.02.

23. Sawkins K, Refshauge K, Kilbreath S, Raymond J. The placebo effect of ankle taping in ankle instability. Med Sci Sports Exerc. 2007;39:781-7.

24. Kunugi S, Masunari A, Yoshida N, Miyakawa S. Postural stability and lower leg muscle activity during a diagonal single-leg landing differs in male collegiate soccer players with and without functional ankle instability. J Phys Fit Sport Med. 2017;6:257-65. https://doi.org/10.7600/jpfsm.6.257.

25. Gribble PA, Delahunt E, Bleakley C, Caulfield B, Docherty C, Fourchet F, et al. Selection criteria for patients with chronic ankle instability in controlled research: A position statement of the international ankle consortium. J Orthop Sports Phys Ther. 2013;43:585-91.

26. Knight AC, Weimar WH. Effects of previous lateral ankle sprain and taping on the latency of the peroneus longus. Sport Biomech. 2012;11:48-56. https://doi.org/10.1080/14763141.2011.637121.

27. Brazen DM, Todd MK, Ambegaonkar JP, Wunderlich R, Peterson C. The effect of fatigue on landing biomechanics in single-leg drop landings. Clin J Sport Med. 2010;20:286-92. https://doi.org/10.1097/JSM.0b013e3181e8f7dc.

28. Feger MA, Donovan L, Hart JM, Hertel J. Effect of ankle braces on lower extremity muscle activation during functional exercises in participants with chronic ankle instability. Int J Sports Phys Ther. 2014;9:476-87. http://www. ncbi.nlm.nih.gov/pubmed/25133076.

29. Cordova ML, Takahashi Y, Kress GM, Brucker JB, Finch AE. Influence of external ankle support on lower extremity joint mechanics during drop landings. J Sport Rehabil. 2010;19:136-48.

\section{Publisher's Note}

Springer Nature remains neutral with regard to jurisdictional claims in published maps and institutional affiliations.

Ready to submit your research? Choose BMC and benefit from:

- fast, convenient online submission

- thorough peer review by experienced researchers in your field

- rapid publication on acceptance

- support for research data, including large and complex data types

- gold Open Access which fosters wider collaboration and increased citations

- maximum visibility for your research: over $100 \mathrm{M}$ website views per year

At $\mathrm{BMC}$, research is always in progress.

Learn more biomedcentral.com/submissions 